\title{
Anesthesia Teaching for Undergraduates; Bringing the Operating Theatre to the Simulation Lab: A Pilot Study
}

\author{
Thiruselvi Subramaniam ${ }^{1,{ }^{*}}$ and Tan Ann Jee ${ }^{2}$ \\ ${ }^{1}$ International Medical University, Seremban, Malaysia \\ ${ }^{2}$ Department of Anaesthesiology, International Medical University, Seremban, Sembilan, Malaysia \\ "Corresponding author: International Medical University, Seremban, Malaysia. Tel: +60-126175335, Email: thiruselvi_subramaniam@imu.edu.my \\ Received 2020 April 11; Accepted 2020 June 28.
}

\begin{abstract}
Background: Simulation-based education has become more prominent in recent years. In our university, we have begun to use simulation at various levels in the curriculum.

Objectives: We decided to utilize simulation to meet the challenges of teaching medical students in the operating theatre, which have slowly risen over the years at our place of practice.

Methods: A total of 120 final year medical students posted to anesthesia were divided into two groups and exposed to two different operating theatre (OT) teaching environments: Group I: Actual OT and group 2: Simulated OT. The performance of the two groups on 25 MCQs was compared using the chi-square test and independent $t$-test.

Results: The chi-square test showed no significant difference between the groups in the number of students who passed the test at the end of the posting $\left[\chi^{2}(2, \mathrm{~N}=119)=2.375, \mathrm{P}>0.05\right]$. An independent $t$-test carried out on the test scores showed that the actual OT group $(M=10.15, S D=2.284)$ performed better than the simulated OT group $(M=9.04, S D=2.528)$, and it was statistically significant $[\mathrm{t}(112)=3.265, \mathrm{P}=0.001]$.

Conclusions: There was no difference in terms of pass or fail between the two groups suggesting that OT learning could be simulated. However, the actual OT group had overall better scores statistically. Thus the depth of learning may be better with actual OT teaching, especially because the teaching and learning occur in the real environment. Until further research suggests that simulated OT can replace actual OT learning for undergraduates, we plan to use the simulated OT as a bridge towards actual OT learning.
\end{abstract}

Keywords: Medical Simulation, Operation Theatre, Clinical Skills, Medical Students, Opportunities, Anesthesia, Simulated Operating Theatre

\section{Background}

Anesthesia is part of the undergraduate curricula in most medical schools, as is in ours, although there appears to be no formal consensus about curriculum content for anesthesia in undergraduate medical education. Teaching in the operating room is the primary teaching method during clinical years in many universities, as this is where the main practice of anesthesia occurs $(1,2)$. The width and depth of content and the technique of delivery appear to vary significantly among institutions though students' exposure and presence in the operating theatre (OT) appear common to all $(1,3-5)$.

Students entering the clinical phase begin with a disadvantage, facing challenges of a change from a relatively safe classroom environment where they are somewhat "spoon-fed" to an environment completely alien to them, the wards, and clinics (6). They are thrown into an environ- ment that expects attitude, behavior, and knowledge of a workplace, which, in a nutshell, is reality. Wards and clinics are benign compared to the entry into the world of OT where the differences are much more, and students struggle navigating through this environment, making sense of OT dressing to OT etiquettes, the dos and don'ts, and more. Whether it is an entry into OT during anesthesia or a surgical-based posting, exploration into students' experiences of learning in the OT indicates common barriers to learning. Attempts have been made to overcome some of these barriers in the form of pre-posting orientation or courses and the use of simulation (6-8).

Medical students' learning in the OT during anesthesia posting has become a challenge at our university in recent years. It has progressively become more difficult to have students spend time learning in the OT due to the increasing number of students from various disciplines 
(nursing and medicine) and different universities utilizing the OT for a similar purpose. Over the years, the headcount has increased with no increase in infrastructure and space, contributing to the challenges. Fear of patient safety due to increased risks of infection has led to the restriction in the number of students allowed to enter into the OT. Hence students are often unable to observe the anesthetic management of a patient from induction to the reversal of anesthesia. They also miss out on the post-operative care of these patients in the recovery room before they are cleared for discharge from OT to the wards. This process is an important component of the anesthesia curriculum.

\section{Objectives}

Our study aimed to identify if the outcome of teaching anesthesia in simulated OT is comparable to the outcome of teaching in the actual OT. This may be a novel way to overcome the problems of undergraduate anesthesia learning at our university.

\section{Methods}

In our curriculum, exposure to anesthesia learning occurs in the final year and over two weeks, linked to four weeks of surgical rotation (a total of six weeks of surgical block posting). Students are expected to go into the OT and observe anesthesia being delivered for all types of surgical procedures.

This is a randomized controlled trial conducted on 120 final year medical students posted to the Department of Anesthesia during their surgical block posting. Students were briefed regarding the study, and written consent was obtained before they were recruited to participate in the study. All students opted to participate in the study though they were given the option to decline. The goals and learning outcomes were decided by the Anesthesia Faculty at the beginning of the semester based on the curriculum. This was to standardize the teaching delivery and content.

The test items were prepared by the Anesthesia Faculty based on the curriculum content. The areas tested included perioperative care, OT processes (patient safety, checklists, consent, emergency, and elective procedures in OT), airway management, basic principles of anesthesia, and monitoring and phases of anesthesia (induction, maintenance, emergence, and recovery care before discharge from OT).

There were a total of 120 students in this final year cohort, and they were divided into 3 groups of 40 students who rotated through 3 major postings; Internal Medicine,
Family Medicine and Surgical block (combined surgery and anesthesia). Each group of 40 students during the surgical block posting were divided into four groups A, B, C, and $D$ and rotated through the Anesthesia and Surgical Department over the six weeks (Figure 1). Randomization of each of the four groups was carried out by having each group's leader to draw lots at the beginning of the posting to decide which session (actual OT/simulated OT) they will be first exposed to. Students in group I initially experienced teaching in the actual OT environment while group II had their initial exposure in the simulated OT.

At the end of week 5, all 40 students completed 25 MCQs. At week 6, all groups were exposed to the simulated or actual OT sessions that they had no experienced initially to ensure that all students were exposed to both types of teaching and learning. The performance of the two groups on the MCQ test was compared using the chi-square test and independent $t$-test.

\subsection{Simulated OT Session}

The simulated OR had a functioning OT table with a high-fidelity manikin on it, a working general anesthesia (GA) delivery machine, and a drug trolley with all drugs and equipment usually found in an actual OR. Clinical skills staff, who were actual nurses, role-played, beginning with the with pass-over from the ward nurse to the OT nurse, which included the communication, checking of the patient using the safe surgery checklists. A precreated scenario of an ASA II (American Society of Anesthesiologists) patient with detailed patient records and consent forms were used. Then, the faculty played the role of an anesthetist and carried out assessments on the patient (standardized patient) before entering the OR. Once in OR, the manikin was used as the patient for further teaching. The processes of checking the machine, equipment, drug preparation, induction, mask ventilation, intubation, monitoring, maintenance, and reversal of anesthesia were carried out. Students got to see physiological changes in blood pressure and heart rate secondary to drugs and intubation as occurs in an actual OT setting. A debriefing session was held at the end of "surgery and anesthesia" to discuss all the intended learning.

\subsection{Actual OT Session}

Students were instructed to enter the OT very early in the morning to observe the exchanges between ward nurses and OT nurses when patients were brought into the OT. Two students were assigned to an OR; hence, they followed the patients' progress from entry into the OT 


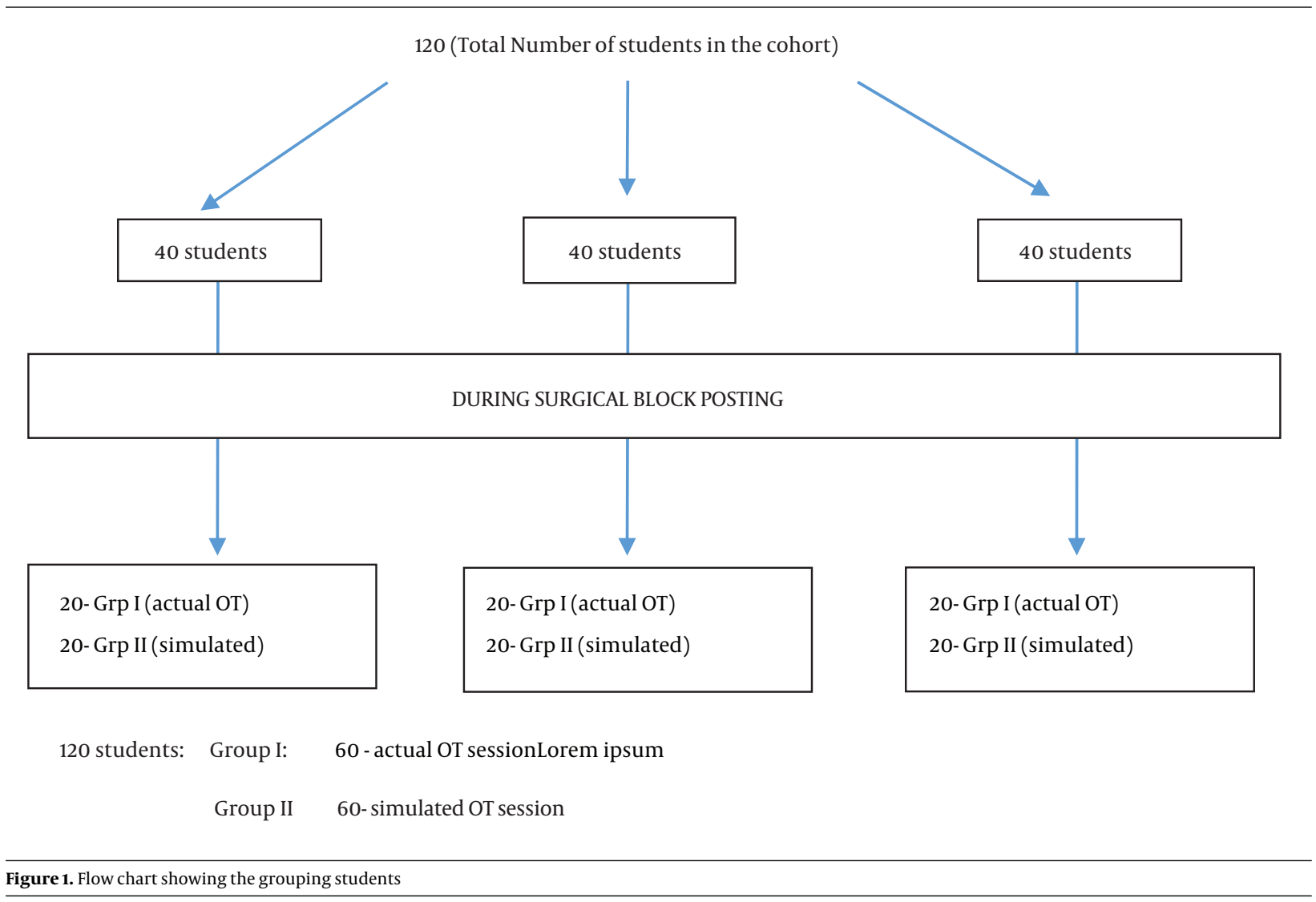

to transfer to the OR. They followed the respective anesthetists to the OR and observed the assessment procedure, patient safety checks, induction, intubation, maintenance, monitoring, and reversal (not always possible as some cases took longer to end). Students, then, presented the cases they had observed to the faculty and had discussions in the recovery area of the OT where patients were observed to avoid grouping in one OR disrupting routine work. At the end of the written test, students were invited to provide verbal feedback regarding their experience with the learning.

\subsection{Statistical Analysis}

An analysis was conducted using SPSS. The performance of the two groups on the MCQ test in terms of scores and pass/fail results were compared using the chi-square test and independent $t$-test. Ethical approval was obtained from the University Ethics Committee before the conduct of research.

\section{Results}

Of the 120 students, one did not participate, and five did not attempt the test as they were absent for the class on the test days; thus, the analysis was performed on 114 students of whom, 49 were males and 65 were females.

Although the number of students who passed the test was slightly higher in group I (actual OT) than in group II (simulated OT), the Chi-square test showed the difference was not significant $\left[\chi^{2}(2, \mathrm{~N}=119)=2.375, \mathrm{P}>0.05\right]$.

The actual OT (group I, $\mathrm{n}=57$ ) had a mean score of 10.51 (SD = 2.284) (Table 1). By comparison, the simulated OT (group II, $\mathrm{n}=57$ ) had a lower mean score $(\mathrm{M}=9.04$; $\mathrm{SD}=2.528$ ). An independent $t$-test to compare the mean scores between the two groups showed that the actual OT group $(M=10.15, S D=2.284)$ performed better than the simulated OT group $(\mathrm{M}=9.04, \mathrm{SD}=2.528)$ and it was statistically significant $[\mathrm{t}(112)=3.265, \mathrm{P}=0.001]$. The actual OT group appeared to obtain better scores than the simulated OT group. 


\begin{tabular}{lcc}
\hline \multicolumn{3}{l}{ Table 1. Descriptive Statistics of Actual and Simulated OT Groups ${ }^{\mathrm{a}}$} \\
\hline Group & Number & Values \\
\hline Actual OT & 57 & $10.51 \pm 2.284$ \\
Simulated OT & 57 & $9.04 \pm 2.528$ \\
\hline
\end{tabular}

${ }^{\mathrm{a}}$ Values are expressed as mean $\pm \mathrm{SD}$.

\section{Discussion}

Awareness of surrounding and learning from experience is well established as an important part of adult learning $(9,10)$. Simulation is becoming a frequently used experiential teaching-learning tool to bridge the gap between the classroom and the actual environment in undergraduate and postgraduate healthcare education (5, 11-14). More reports on the benefits of simulation in clinical education are accumulating, validating its relevance in the healthcare industry. The need to expand and explore the use of simulation in a more diverse manner is still lacking for various reasons. Simulation-based teaching is very resourceintensive, and the preparation for the teaching is sometimes longer than the conduct of the simulated session per se. That and the lack of resources can make simulated teaching more challenging for faculties who are not proponents of this style of teaching in the first place.

The needs assessment and feedback from students about the challenges facing when attending the actual OT spurred the decision to attempt the inclusion of simulated OT teaching as part of our anesthesia teaching delivery style. Students' first exposure to OT set up is when they enter the clinical phase in year 3 and start their surgical based rotations. At this point, they enter the theatre to observe the surgical cases and do not get any exposure on anesthesia provided for the patients as it is not part of their year 3 curriculum. At the beginning of the surgical posting, they are briefed about the OT etiquettes and processes by surgical faculty before they venture into the OT. However, the quality of learning is threatened by the lack of knowledge about the basic functioning and system processes of an OT. The OT can be quite overwhelming to first-timers as it is usually a very busy zone with constantly changing situations. Students observe the environment, staff in action, activities, monitoring, and exchanges without really understanding the important nuances that are relevant to their actual learning. These are "hidden" areas of learning that they are not aware of and where a prior exposure in a simulated setting would help shed some light and create awareness, allowing faster acclimatization to the OT environment, making understanding and learning in the actual environment more constructive (6). The group of stu- dents in our research was in year 5 and had been to the OT during their surgical posting in year 3 but without exposure to anesthesia. Having said that, the results appeared to indicate the lack of knowledge in both groups under study, perhaps indicating a need to pause and rethink or restrategize our teaching. Most in both groups did not perform well in the MCQ test.

Knight et al. (6), identifying many problems in the OT, conducted an induction course for students before anesthesia posting to overcome many of these issues. They had positive feedback from students who felt they were more confident in the theatre after the course, which goes to imply that some exposure before the actual learning environment has some benefits. A study conducted in a simulated OT environment to orientate third-year medical students entering the surgery clerkship for the first time had positive feedback on the learning experience. Students reported being more confident when in the OR and better understand how they could become more involved on their first day there (8). A literature review of medical student learning in the theatre environment found several common themes that affected their learning. Students described the lack of clear objectives, fear, anxiety, and feelings of humiliation and intimidation as some of the barriers to their OT learning. The study identified concerted effort towards the preparation of students for theater setting as one of the ways to overcome some barriers to OT learning (15). Similarly, another study by Bowrey and Kidd (16) looked at early emotional experiences in the OT and their influences on medical student learning; all participants reported initial negative emotions (apprehension, anxiety, fear, shame, overwhelmed), with negative feelings like loss of familiarity, organizational issues, and concerns about violating the protocol. Their early learning experiences centered around adjustment to the physical environment of the OT, and most students needed an average of one week to overcome these barriers before they could be comfortable and learn better (16). These studies further enforce the need for some form of pre-OT exposure before entry to the actual environment.

Students reported a positive learning experience with simulators and an opportunity to apply their knowledge in a realistic environment in research done by Cleave-Hogg and Morgan (17) Our students also responded that they found the simulated session to be helpful before the actual session as they could better appreciate and be aware of the happenings in the OT.

A literature search for similar research was not successful though we did find a related study that compared simulated teaching with didactic teaching (18). In this study, the 
authors noted that though there was greater improvement in post-teaching MCQ among the simulator group, baseline scores were higher in the lecture group. In our study, we also noted that though there was no statistical difference in the result of the test between the groups in the numbers that passed or failed the MCQ test, the actual OT group had better scores. However, both groups did not do well in MCQs, which, in itself, questions the teaching and how much students actually learn during their sessions in the OT setting. A study that compared the effectiveness of teaching general anesthesia induction using simulation and traditional supervised teaching found that the simulation group performed better in $25 \%$ of tasks than the traditional group (19). Training for surgical specialists on crisis resource management (CRM) in a simulated OT set up at some centers showed benefits (20,21). Extrapolating from this, undergraduate teaching may benefit from the simulation as a teaching tool, especially for experiential learning.

The extent to which students become immersed in a simulated clinical scenario (degree of immersiveness) can be improved by increasing the fidelity. Students then become engaged psychologically and view the situation they are in as real, becoming the person they represent in the scenario.

Recreating an OR environment that closely resembles the actual OR and using a standardized patient(SP), changing into OT scrubs were techniques we utilized to enhance the fidelity of the scenario. We utilized a high-fidelity manikin in the simulated OR environment, attempting to simulate the actual OR as we best could, given the resources and environment. The OT reception bay was a corridor outside the simulated OR that was cordoned off to create an enclosed area. Many factors could have influenced our research as simulating some aspects of an actual OT situation was not possible, especially the hustle and bustle of the real OT with the inter-professional team environment.

During debriefing and feedback sessions, however, the response to the simulated OT sessions was positive and well received by students. Students felt that they were more aware of the roles of OT personnel, patient safety processes involved, and perioperative care of patients. A more immersive simulated experience created by a more realistic environment that includes a multi-professional team and a relook at the learning outcomes and what students actually perceive may help improve their performance after the exposure. A major challenge would arguably be the availability of resources and time constraints.

\subsection{Limitations}

The immersiveness of the students due to the lack of a multi-professional team (nurses, surgeons, other OT support staff).

Using test questions not validated by other anesthetists outside the university.

The fidelity of the environment.

Individual student's intellectual capacity and preparation before classes.

The conduct of the sessions by two instructors instead of the same person for all the groups.

Using unequal time intervals between teaching sessions and the test for all the groups due to the logistics of students' schedules.

\subsection{Conclusions}

While we acknowledge the limitations of our study, we also interpret the results in a positive light, even as encouraging in that, there was no statistical difference in the outcome of both the teaching styles in terms of the number of students who passed or failed the test though there were differences in actual scores, suggesting the possibility of a future for OT teaching in the simulated OT environment even if it is just as a bridge to help transfer skills to be performed in the actual environment.

However, whether it can replace actual OT learning is not answered here though similar research in the future may provide more information and answers. This research has, in some ways, been an eye-opener for the faculty as it affirms the difficulty in predicting what students actually learn no matter how well we design our instructions. At our university, the introduction to simulated OT environment at the beginning of the clinical phase may help students adapt to face the complexities of the actual "alien" OT environment and improve learning. At the very least, we now look towards using the simulated OT as a path towards improved learning experiences in the actual environment.

\section{Footnotes}

Authors' Contribution: None declared by author.

Conflict of Interests: There exist no conflicts of interest due to financial and personal relationships.

Ethical Approval: Ethical approval was obtained from the University Ethics Committee before the conduct of the research.

Funding/Support: There was no funding required for this research. 
Informed Consent: Students were briefed regarding the study, and written consent was obtained before they were recruited to participate in the study.

\section{References}

1. Rohan D, Ahern S, Walsh K. Defining an anaesthetic curriculum for medical undergraduates. A Delphi study. Med Teach. 2009;31(1):e1-5. doi: 10.1080/01421590802334291. [PubMed: 19253149].

2. Curry SE. Teaching medical students clinical anesthesia. Anesth Analg. 2018;126(5):1687-94. doi: 10.1213/ANE.0000000000002802. [PubMed: 29401078].

3. Sidhu NS, Weller JM, Mitchell SJ. Teaching and learning in undergraduate anaesthesia: A quantitative and qualitative analysis of practice at the University of Auckland. Anaesth Intensive Care. 2015;43(6):7409. doi: 10.1177/0310057X1504300612. [PubMed: 26603799].

4. Cheung V, Critchley LA, Hazlett C, Wong EL, Oh TE. A survey of undergraduate teaching in anaesthesia.Anaesthesia.1999;54(1):4-12. doi: 10.1046/j.1365-2044.1999.00640.x. [PubMed: 10209363].

5. Fodale V, Penna O, Amato A, Bruno R, Tripodi VF, Vadalà EG, et al. Role of advanced simulation in undergraduate and postgraduate medical education. Int Arch Med. 2015. doi: 10.3823/1695.

6. Knight WRC, Glaysher MA, Wheble GAC. Introduction to theatre and basic surgical and anaesthetic skills: A new theatre induction course for medical students. Bull Royal Coll Surg Engl. 2013;95(1):1-4. doi: 10.1308/147363513x13500508916892.

7. Lyon PM. Students' experiences of learning in the operating theatre. Sydney: Sydney University Press; 2007.

8. Barnum TJ, Salzman DH, Odell DD, Even E, Reczynski A, Corcoran J, et al. Orientation to the Operating Room: An introduction to the surgery clerkship for third-year medical students. MedEdPORTAL. 2017;13:10652. doi: 10.15766/mep_2374-8265.10652. [PubMed: 30800853]. [PubMed Central: PMC6338138].

9. Kelly C. David Kolb, the theory of experiential learning and ESL. Internet TESL J.1997;3(9):1-5.

10. Kolb DA. Experiential learning: Experience as the source of learning and development. New Jersey(US): FT press; 2014

11. Al-Elq AH. Simulation-based medical teaching and learning. J Family Community Med. 2010;17(1):35-40. doi: 10.4103/1319-1683.68787. [PubMed: 22022669]. [PubMed Central: PMC3195067].
12. Motola I, Devine LA, Chung HS, Sullivan JE, Issenberg SB. Simulation in healthcare education: A best evidence practical guide. AMEE Guide No. 82. Med Teach. 2013;35(10):e1511-30. doi: 10.3109/0142159X.2013.818632. [PubMed: 23941678].

13. O'Flynn S, Shorten G. Simulation in undergraduate medical education. Eur $J$ Anaesthesiol. 2009;26(2):93-5. doi: 10.1097/EJA.0b013e32831a47df. [PubMed: 19142080].

14. Supramaniam PR, Mittal M, Davies R, Lim LN, Arambage K. Didactic lectures versus simulation training: A randomised pilot evaluation of its impact on surgical skill. Gynecol Surg. 2018;15(1). doi:10.1186/s10397018-1053-5.

15. Croghan SM, Phillips C, Howson W. The operating theatre as a classroom: A literature review of medical student learning in the theatre environment. Int J Med Educ. 2019;10:75-87. doi: 10.5116/ijme.5ca7.afd1. [PubMed: 31012867]. [PubMed Central: PMC6766388].

16. Bowrey DJ, Kidd JM. How do early emotional experiences in the operating theatre influence medical student learning in this environment? Teach Learn Med. 2014;26(2):113-20. doi 10.1080/10401334.2014.883986. [PubMed: 24702546].

17. Cleave-Hogg D, Morgan PJ. Experiential learning in an anaesthesia simulation centre: analysis of students' comments. Med Teach. 2002;24(1):23-6. doi: 10.1080/00034980120103432. [PubMed: 12098453].

18. Solymos O, O'Kelly P, Walshe CM. Pilot study comparing simulationbased and didactic lecture-based critical care teaching for final-year medical students. BMC Anesthesiol. 2015;15:153. doi: 10.1186/s12871-0150109-6. [PubMed: 26490826]. [PubMed Central: PMC4617911].

19. Hallikainen J, Vaisanen O, Randell T, Tarkkila P, Rosenberg $\mathrm{PH}$ Niemi-Murola L. Teaching anaesthesia induction to medical students: Comparison between full-scale simulation and supervised teaching in the operating theatre. Eur J Anaesthesiol. 2009;26(2):101-4. doi: 10.1097/EJA.ob013e32831a6a76. [PubMed: 19142082].

20. Aggarwal R, Undre S, Moorthy K, Vincent C, Darzi A. The simulated operating theatre: Comprehensive training for surgical teams. Qual SafHealth Care. 2004;13 Suppl 1:i27-32. doi: 10.1136/qhc.13.suppl_1.i27. [PubMed: 15465952]. [PubMed Central: PMC1765789]

21. Kieu V, Stroud L, Huang P, Smith M, Spychal R, Hunter-Smith D, et al. The operating theatre as classroom: A qualitative study of learning and teaching surgical competencies. Educ Health (Abingdon). 2015;28(1):22-8. doi: 10.4103/1357-6283.161845. [PubMed: 26261110]. 